\title{
Renormalization Group Properties of Higher-Derivative Quantum Gravity with Matter in $4-\varepsilon$ Dimensions
}

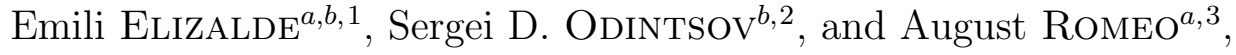 \\ ${ }^{a}$ Centre for Advanced Studies CEAB, CSIC, Camí de Santa Bàrbara, 17300 Blanes \\ ${ }^{b}$ Department ECM and IFAE, Faculty of Physics, University of Barcelona, \\ Diagonal 647, 08028 Barcelona, Catalonia, Spain
}

\begin{abstract}
We investigate the phase structure and the infrared properties of higher-derivative quantum gravity (QG) with matter, in $4-\varepsilon$ dimensions. The renormalization group (RG) equations in $4-\varepsilon$ dimensions are analysed for the following types of matter: the $f \varphi^{4}$-theory, the $O(N) \varphi^{4}$ theory, scalar electrodynamics, and the $S U(2)$ model with scalars. New fixed points for the scalar coupling appear - one of which is IR stable - being some of them induced by QG. The IR stable fixed point perturbed by QG leads to a second-order phase transition for the theory at non-zero temperature. The RG improved effective potential in the $S U(2)$ theory (which can be considered as the confining phase of the standard model) is calculated at nonzero temperature and it is shown that its shape is clearly influenced by QG.
\end{abstract}

\footnotetext{
${ }^{1}$ E-mail: eli@zeta.ecm.ub.es

${ }^{2}$ On leave of absence from Tomsk Pedagogical Institute, 634041 Tomsk, Russia. E-mail: odintsov@ecm.ub.es

${ }^{3}$ E-mail: august@ceab.es, august@zeta.ecm.ub.es
} 


\section{Introduction}

The infrared (IR) structure of quantum gravity (QG) is very important in the applications of QG to particle physics and cosmology at scales below the Planck mass. Regarded from another side, the IR behaviour of QG is closely connected with its thermal properties. For example, the study of Einstein gravity on a de Sitter background [1] is motivated not only by possible cosmological applications, but also by the need to understand the IR structure of the graviton propagator [2]. IR dynamics of QG is most probably relevant to the solution of the cosmological constant problem [3]. Further exploiting this point of view, an effective theory [4] based on integrated conformal anomaly dynamics [5] has been developed, aimed at the description of QG in the far infrared region. Such a model may provide a very natural solution of the cosmological constant problem.

To investigate the IR properties of a theory, the well-known $\varepsilon$-expansion technique is often very useful, as it happens to be the case in the theory of critical phenomena (see [6]- [8] for an introduction). For renormalizable theories, this technique supplies a quite standard way of studying the critical behaviour and the nature of its phase-transitions at nonzero temperature [9, 10]. (A discussion on the equivalence between the IR behaviour of finite-temperature theories and three-dimensional theories can be found in [11, 12]).

In $\mathrm{QG}$, the $\varepsilon$-expansion technique has been applied to study the corresponding $(2+\varepsilon)$ dimensional theory [13, 14, 15]. However, standard Einstein gravity is not renormalizable, not even in $2+\varepsilon$ dimensions 14 . To overcome this difficulty, it looks certainly interesting to study a multiplicatively renormalizable QG starting from a different standpoint in $D=4-\varepsilon$ dimensions. Such a study is expected to be a more realistic approach to QG than the $D=2+\varepsilon$ theory, since two-dimensional theories have very specific properties.

At the present moment there is no completely consistent theory of quantum gravity. Standard Einstein gravity — which is very fine as a classical theory — is regretfully non-renormalizable [16]. String theory (see [17] for a general review) may certainly lead to an effective gravity, which in general should also be considered at the classical level (being also, as a rule, nonrenormalizable). Under such circumstances, there is still the following possibility to take into account quantum gravity effects: to work with some effective theory of QG which mimics some of the essential properties of the fully consistent theory. Basically one can think of such an effective theory as of an expansion of the (unknown) gravitational Lagrangian in powers of curvature invariants. In principle such a theory will be, generically speaking, non-renormalizable. One exceptional example is, however, four-dimensional higher-derivative gravity, which is known to be multiplicatively renormalizable [18] (in a scheme, of course, of a fourth-order propagator). Moreover, this theory is asymptotically free [19]-21], and has Einstein's theory as a low-energy limit (hence, it is undistinguishable from Einstein's theory at the classical level). Owing to the fact that the gravitational couplings in such a theory are dimensionless, they might show up in different situations of high-energy physics, in the standard description of quantum field theory.

This theory of QG is certainly not free from its own problems, what has ruled it out from the very short list of candidates for describing $Q G$ in a fundamental way. The main difficulty associated with any model of higher-derivative QG is perhaps the unitarity problem 
at the perturbative level. However, a real chance of solving the unitarity problem in a nonperturbative approach exists (see, for example, the paper by Antoniadis and Tomboulis in Ref. [20]). One has to emphasize that the problem of non-unitarity is of dynamical nature, so that one is compelled to consider it with account of all kind of quantum corrections (perturbative and non-perturbative ones). In this respect, the string example [22] - where it happens that negative-norm states decouple at the renormalization group (RG) fixed point - may be a good lesson to learn. Also, when considered as some sort of effective theory - that ought to be replaced with the true fundamental theory of QG at high energies - the loss of unitarity should not be the ultimate argument against higher-derivative gravity.

When considered in the energy region between the GUT scale and the Planck scale, and in interaction with some asymptotically-free GUT, higher-derivative gravity does not, as a rule, destroy asymptotical freedom and, hence, a totally asymptotically free theory which represents the unification of $R^{2}$-gravity with a GUT [19] can be easily constructed. Then, one sees the appearance of one-loop quantum gravity corrections to scalar and Yukawa couplings, what may influence the GUT in different respects. In the present paper - motivated in addition by the big activity that is going on about the study of $(2+\epsilon)$-dimensional gravity - we suggest investigating the influence of the above mentioned QG corrections on the phase transitions that occur in scalar-gauge theories, and in their infrared properties, working with the $\epsilon$-expansion technique [7] near four dimensions. Finally, even if one would still insist upon the point that higher-derivative gravity is not very realistic on some ground or other (as we have argued before it may have some relevance to reality), the theory to be considered here constitutes a rather nice and completely workable example where, as we shall see, the critical dynamics can be studied up to almost the same level as in the absence of QG, what is already a quite appealing issue (for a list of different theories whith similar studies of their properties in relation with critical phenomena, see [6]).

The paper is organized as follows. In the next section we give the action of QG with matter in $4-\varepsilon$ dimensions, and analyse some of their properties. In section 3 we investigate the $\mathrm{RG}$ equations in the case of higher-derivative QG interacting with matter in $4-\epsilon$ dimensions. One of the main motivations for such a study is, of course, the search for finite-temperature phase transitions and, hence, we would wish to set $\epsilon=1$ in the end. Unfortunately, in general the $\epsilon$ expansion [7] works well only near $D=4$, i.e., for infinitesimal $\epsilon$. As usual in these cases, we will here assume that the results rigorously derived for small $\epsilon$ continue to be valid at $\epsilon=1$. In other areas of physics there exist plenty of explicit examples (see, for instance, Ref. [6]) where, having at our disposal both numerical and experimental data, the comparison of both gives unexpectedly good agreement (especially for the case of second order phase transitions). Regretfully, in the theory under consideration here we have yet no numerical results to compare, for simulations of QG are very hard to do, even in low dimensions (see Ref. [23] for a recent review). But, from the mentioned experience it would come as no surprise if things would really match again, when the comparison can be established. The general structure of the solutions and the appearance of new fixed points induced by QG (in the case of the $f \varphi^{4}$-theory) are discussed, as well as their relevance to the study of QG at nonzero temperature. Note that in earlier papers (see [24, 25] and references therein) mainly Einstein QG at nonzero temperature 
has been studied. In particular, the temperature corrections to the graviton amplitudes have been discussed in Ref. [25]. We will also investigate the fact that near the IR stable fixed point a second order phase transition occurs. In the case of the $S U(2)$ model, only unstable fixed points exist (due to the fact that $g^{* 2}=0$ ) and hence a first order phase transition is to be expected.

Section 4 is dedicated to the study the RG equations in higher-derivative QG with the $O(N)$ model. The phase structure is numerically investigated, showing that at large $N$ the explicit value of the fixed point decreases. In section 5 we study the RG-improved effective potential at nonzero temperature in a $S U(2)$ model with $\mathrm{QG}$ and estimate the influence of $\mathrm{QG}$ on the confining phase of the standard model (SM). We conclude with an overall discussion and an outlook.

\section{Higher-derivative QG with matter in $4-\varepsilon$ dimensions}

We will be interested in studying the behaviour of theories in $4-\varepsilon$ dimensions (eventually $\varepsilon$ will be chosen to be 1) with a Lagrangian of the form

$$
\mathcal{L}=\mu^{-\varepsilon}\left(\frac{1}{2 \lambda} W-\frac{\omega}{3 \lambda} R^{2}+\chi R+\Lambda\right)+\frac{1}{2} \xi R \varphi^{2}+\mathcal{L}_{m}\left(\varphi, A_{\mu}^{a}\right),
$$

where $W=C_{\mu \nu \alpha \beta} C^{\mu \nu \alpha \beta}$ is the square of the Weyl tensor, $\varphi$ is a scalar and $\mu$ is a mass parameter which is used to make $\lambda$ dimensionless in $4-\varepsilon$ dimensions. The symbolic form for the matter Lagrangian is

$$
\mathcal{L}_{m}\left(\varphi, A_{\mu}^{a}\right)=-\frac{1}{4} G_{\mu \nu}^{a} G^{a \mu \nu}+\frac{1}{2} g^{\mu \nu}\left(\nabla_{\mu} \varphi\right)^{a}\left(\nabla_{\nu} \varphi\right)^{a}-\frac{1}{2} m^{2} \varphi^{2}-\frac{1}{4 !} f \mu^{\varepsilon} \varphi^{4},
$$

where $\varphi^{2}=\varphi^{a} \varphi^{a},\left(\nabla_{\mu} \varphi\right)^{a}$ is a covariant derivative in which the standard gauge coupling $g$ has been changed in the way $g \rightarrow \mu^{\varepsilon / 2} g$, in order to keep $g$ dimensionless for the number of dimensions $D=4-\varepsilon$. The same is true for $f$.

To study the critical behaviour of such a system, which includes a higher-derivative QG sector plus the (non)-abelian Higgs model (for an introduction to critical phenomena, see [6][8]), it is enough to consider the renormalizable subset of the theory 10 where masses are extremely small or zero. So, we may put $m^{2}=0$ in (2.2). We shall later discuss the different content of fields and different gauge groups in $\mathcal{L}_{m}(2.2)$.

It is worth noting that a detailed introduction to higher-derivative QG (with matter) was given in the book [19] (see also refs. therein). As mentioned in the introduction, such a theory is known to be multiplicatively renormalizable in $D=4$ and also asymptotically free for all coupling constants. Asymptotic freedom in the matter sector may be also realized [19, 21] (if the gauge couplings show an AF behaviour, i.e. if abelian vectors are not included in the theory), hence the QG couplings play in some sense the role of a Yukawa coupling constant.

The study of the RG behaviour of the dimensional parameters $(\chi, \Lambda)$ - which play the role of some kind of mass for the gravitational field - is of less interest to us. First of all, the 
RG equations for these couplings are gauge dependent and, in order to render them gaugeindependent, one has to introduce some combination (an essential coupling constant). The RG equation for this essential coupling constant is certainly gauge-independent. Moreover, without the cosmological and Einstein terms, the theory (2.1) is multiplicatively renormalizable if the scalar mass in (2.2) is zero. Applying the same arguments as in 19, 10] - namely that in order to study its critical behaviour it is enough to consider the effective renormalizable subset of the theory with zero masses (which is indeed the case!) - one can actually drop these terms from the very beginning. So, we shall concentrate only on the study of the terms which have dimensionless coupling constants in four dimensions.

It is interesting to observe that even if one starts from the theory without Einstein or cosmological terms, one may recover them in the low-energy theory as a result of spontaneous symmetry breakdown (or a curvature-induced phase transition [26]). Note also that in what follows we assume a topologically trivial, flat background for the gravitational field.

\section{Renormalization group equations for $R^{2}$-gravity with matter}

In this section we will consider the $R G$ equations using the $\varepsilon$-expansion analysis. As specific examples, we take a few different matter theories, in particular the $f \varphi^{4}$-theory, scalar electrodynamics, and the nonabelian $S U(2)$ model. Observe that we will work in frames of standard perturbation theory, where QG corrections are essential.

Let us consider the $f \varphi^{4}$-theory in flat $D=4-\varepsilon$ dimensional spacetime. Then,

$$
\mathcal{L}_{m}=\frac{1}{2} \partial^{\mu} \varphi \partial_{\mu} \varphi-\frac{1}{4 !} f \mu^{\varepsilon} \varphi^{4}
$$

As usual, in $4-\varepsilon$ dimensions the RG flows are generated by the RG equation:

$$
\frac{d f}{d t}=-\varepsilon f+\beta_{f}(f)
$$

where $\beta_{f}$ is the four-dimensional $\beta$-function in the theory (3.1) and we are interested in its $t \rightarrow-\infty$ behaviour. Indeed, it is the IR behaviour of the theory what dertermines the critical features of the system. The one-loop $\beta$-function for the $f \varphi^{4}$-theory is well known:

$$
\beta_{f}=\frac{3 f^{2}}{(4 \pi)^{2}}
$$

As one can see, apart from $f^{*}=0$ - which is unstable - there is a stable fixed point at

$$
f^{*}=\frac{(4 \pi)^{2} \varepsilon}{3}
$$


(of course, the value of $f^{*}$ is quite large). This is a quite known result (see, for example [8, [6) which shows that such a model near a phase transition gives a second-order transition behaviour. The solution of (3.2) may be explicitly written:

$$
f(t)=\frac{f \varepsilon(4 \pi)^{2}}{3 f-\left(3 f-\varepsilon(4 \pi)^{2}\right) e^{\varepsilon t}} .
$$

Clearly, as $t \rightarrow-\infty, f(t) \rightarrow f^{*}$. As is well known, first order phase transitions are very common in quantum field theory. We will also find that, in the system under discussion here, mainly first order phase trasitions occur but, in some cases we will obtain second order ones, what will be certainly a nice result. In fact, first order phase transitions are of lesser physical interest, in the mean field theory language [6]: the correlation length corresponding to such systems is finite and no universality emerges. As it was mentioned long ago [10], if the theory possesses no stable fixed point in the $\epsilon$ expansion then the first order phase transitions that are predicted are, in general, only weakly of first order (some other effects can become important, as well). Concerning the more physically interesting second order phase transitions, they are not so common, as mentioned already. In the $\epsilon$-expansion technique we are mainly interested in $\epsilon=1$, i.e. in finite temperature systems. And it was pointed out in [6]- 8 that for second order phase transitions the lowest order results at $\epsilon$, often agree very well with the experiments (even at $\epsilon=1$ ), when they are available.

The first example will be scalar self-interacting theory with higher derivative gravity. In this case the system of RG equations is

$$
\begin{aligned}
\frac{d \lambda}{d t}=\beta_{\lambda} & =-\varepsilon \lambda-\frac{799 \lambda^{2}}{60(4 \pi)^{2}}, \\
\frac{d \omega}{d t}=\beta_{\omega} & =-\frac{\lambda}{(4 \pi)^{2}}\left[\frac{10}{3} \omega^{2}+\left(18+\frac{19}{60}\right) \omega+\frac{5}{12}+\frac{3}{2}\left(\xi-\frac{1}{6}\right)^{2}\right], \\
\frac{d \xi}{d t}=\beta_{\xi} & =\frac{1}{(4 \pi)^{2}}\left[f\left(\xi-\frac{1}{6}\right)+\lambda \xi\left(-\frac{3}{2} \xi^{2}+4 \xi+3+\frac{10}{3} \omega-\frac{9}{4 \omega} \xi^{2}+\frac{5}{2 \omega} \xi-\frac{1}{3 \omega}\right)\right] \\
& \equiv \beta_{\xi}^{(0)}+\Delta \beta_{\xi}, \\
\frac{d f}{d t}=\beta_{f} & =-\varepsilon f+\frac{1}{(4 \pi)^{2}}\left[3 f^{2}+\lambda^{2} \xi^{2}\left(15+\frac{3}{4 \omega^{2}}-\frac{9 \xi}{\omega^{2}}+\frac{27 \xi^{2}}{\omega^{2}}\right)\right. \\
& \left.\quad-\lambda f\left(5+3 \xi^{2}+\frac{33}{2 \omega} \xi^{2}-\frac{6}{\omega} \xi+\frac{1}{2 \omega}\right)\right] \\
& \equiv-\varepsilon f+\beta_{f}^{(0)}+\Delta \beta_{f},
\end{aligned}
$$

where $\beta_{f}^{(0)}=\frac{3 f^{2}}{(4 \pi)^{2}}, \beta_{\xi}^{(0)}=\frac{f\left(\xi-\frac{1}{6}\right)}{(4 \pi)^{2}}$, the remaining parts of $\beta_{\xi}$ and $\beta_{f}$ defining $\Delta \beta_{\xi}$ and $\Delta \beta_{f}$, which are universal QG corrections to $\beta_{\xi}$ and $\beta_{f}$.

For $\lambda>0$ (this is the situation that is usually considered, and then the classical action is positively defined) we have asymptotic freedom in 4 dimensions. In this case, as in ordinary gauge theories [10], the only fixed point is $\lambda^{*}=0$. Moreover, for $0<\lambda<1$ the IR trajectories will always go away from $\lambda^{*}=0$, what is an indication of a first-order phase transition in such theory. 


\begin{tabular}{c|r|r|r|r|c|} 
& $\lambda$ & $\omega$ & $\xi$ & $f$ & nature \\
\hline 1 & -11.858 & -0.298 & -1.612 & 278.596 & saddle point \\
2 & -11.858 & -0.025 & 0 & 0 & $"$ \\
3 & -11.858 & -0.023 & 0.201 & 17.336 & $"$ \\
4 & -11.858 & -0.023 & 0.203 & 9.806 & IR stable fixed point \\
5 & -11.858 & -5.470 & 0 & 0 & saddle point \\
6 & -11.858 & -5.470 & 0.026 & 33.108 & $"$ \\
\hline 1 & 0 & arbitrary & arbitrary & 0 & unstable point \\
2 & 0 & arbitrary & $\frac{1}{6}$ & $\frac{\varepsilon(4 \pi)^{2}}{3}$ & $"$ \\
\hline
\end{tabular}

Table 1: Numerical values for the fixed points of the RG equations (3.6) (for $\varepsilon=1$ ).

However, taking into account that we are interested in the IR phase of the model, where the properties of the classical action are not important, we may choose also $\lambda<0$ [32]. In this case, we have the fixed point

$$
\lambda^{*}=-\frac{60 \varepsilon(4 \pi)^{2}}{799} .
$$

The general solution is

$$
\lambda(t)=\frac{\lambda e^{-\varepsilon t}}{1-\frac{799 \lambda}{60 \varepsilon(4 \pi)^{2}}\left(e^{\varepsilon t}-1\right)},
$$

which again shows the existence of the fixed point (3.7) in the IR $(t \rightarrow-\infty)$.

Table 1 is obtained by numerically solving equations (3.6) with the r.h.s. set to zero. There is only one IR stable fixed point. Notice that the value of $f$ corresponding to the stable fixed point is of the same order as - but significantly smaller than - in the absence of QG. The remaining five fixed points are saddle points (this is seen after careful numerical analysis of the matrix of derivatives of the beta functions). Thus, we observe that there exists an IR stable fixed point at which a second order phase transition is taking place. Moreover, we get a richer phase structure (four new fixed points) as compared with the case of no QG.

Let us consider now scalar QED

$$
\begin{aligned}
\mathcal{L}_{m}= & \frac{1}{2}\left(\partial_{\mu} \varphi_{1}-e \mu^{\varepsilon / 2} A_{\mu} \varphi_{2}\right)^{2}+\frac{1}{2}\left(\partial_{\mu} \varphi_{2}-e \mu^{\varepsilon / 2} A_{\mu} \varphi_{1}\right)^{2} \\
& +\frac{\xi}{2} R \varphi^{2}-\frac{1}{4 !} f \mu^{\varepsilon} \varphi^{4}-\frac{1}{4} F_{\mu \nu}^{2},
\end{aligned}
$$


where $\varphi^{2}=\varphi_{1}^{2}+\varphi_{2}^{2}$. One can write the RG equations for scalar QED with $R^{2}$-gravity as:

$$
\begin{aligned}
\frac{d \lambda}{d t} & =\beta_{\lambda}=-\varepsilon \lambda-\frac{203 \lambda^{2}}{15(4 \pi)^{2}}, \\
\frac{d e^{2}}{d t} & =\beta_{e^{2}}=-\varepsilon e^{2}+\frac{2 e^{4}}{3(4 \pi)^{2}}, \\
\frac{d \omega}{d t} & =\beta_{\omega}=-\frac{\lambda}{(4 \pi)^{2}}\left[\frac{10}{3} \omega^{2}+\left(5+\frac{203}{15}\right) \omega+\frac{5}{12}+3\left(\xi-\frac{1}{6}\right)^{2}\right], \\
\frac{d \xi}{d t} & =\beta_{\xi}=\frac{1}{(4 \pi)^{2}}\left(\xi-\frac{1}{6}\right)\left(\frac{4}{3} f-6 e^{2}\right)+\Delta \beta_{\xi}, \\
\frac{d f}{d t} & =\beta_{f}=-\varepsilon f+\frac{1}{(4 \pi)^{2}}\left(\frac{10}{3} f^{2}-12 e^{2} f+36 e^{4}\right)+\Delta \beta_{f},
\end{aligned}
$$

where $\Delta \beta_{\xi}$ and $\Delta \beta_{f}$ are defined in (3.6). For $\lambda<0, e^{* 2}=\frac{3}{2} \varepsilon(4 \pi)^{2}$ the numerical solution of the system for the fixed points shows no fixed point for $f$. When $e^{* 2}=0, \lambda^{*}=0$, the situation is qualitatively the same as in pure $f \varphi^{4}$-theory with $R^{2}$-gravity - we get a few unstable fixed points for $f$.

Now, we will discuss the $S U(2)$ gauge theory with scalars (in the adjoint representation of the gauge group. Such a model is of importance because the IR behaviour of the SM at finite temperature is described by the confining phase of some $S U(2)$ gauge theory with scalars. Note that this theory is asymptotically free (see [34, 35]).

The Lagrangian we are going to study is given by

$$
\mathcal{L}_{m}=-\frac{1}{4} G_{\mu \nu}^{a 2}+\frac{1}{2}\left(\nabla_{\mu}^{a b} \varphi_{b}\right)^{2}-\frac{f \mu^{\varepsilon}}{4 !}\left(\varphi_{a}^{2}\right)^{2}+\frac{\xi}{2} R \varphi_{a}^{2}
$$

where $\nabla_{\mu}^{a b}=\delta^{a b} \nabla_{\mu}+\mu^{\varepsilon / 2} g \epsilon^{a c b} A_{\mu}^{c}, a=1,2,3$. This theory, including also spinors, was studied in flat and curved spacetime in the AF regime in refs. [30] and [31, respectively.

In the case of the $S U(2)$ theory, we get

$$
\begin{aligned}
& \frac{d \lambda}{d t}=\beta_{\lambda}=-\varepsilon \lambda-\frac{279 \lambda^{2}}{20(4 \pi)^{2}} \\
& \frac{d g^{2}}{d t}=\beta_{g^{2}}=-\varepsilon g^{2}-\frac{14 g^{4}}{(4 \pi)^{2}} \\
& \frac{d \omega}{d t}=\beta_{\omega}=-\frac{\lambda}{(4 \pi)^{2}}\left[\frac{10}{3} \omega^{2}+\left(5+\frac{279}{20}\right) \omega+\frac{5}{12}+\frac{9}{2}\left(\xi-\frac{1}{6}\right)^{2}\right] \\
& \frac{d \xi}{d t}=\beta_{\xi}=\frac{1}{(4 \pi)^{2}}\left(\xi-\frac{1}{6}\right)\left(\frac{5}{3} f-8 g^{2}\right)+\Delta \beta_{\xi}, \\
& \frac{d f}{d t}=\beta_{f}=-\varepsilon f+\frac{1}{(4 \pi)^{2}}\left(\frac{11}{3} f^{2}-24 g^{2} f+72 g^{4}\right)+\Delta \beta_{f} .
\end{aligned}
$$

The corresponding system for the fixed points does not have a stable solution. Only the unstable fixed points $\lambda^{*}=0$ (when QG effects effectively disappear) or $g^{2 *}=0, \lambda^{*}<0$, and some other associated fixed points for $f$. 
A few words about critical exponents are called for. For $R^{2}$-gravity with matter we have few mass-like terms; so, in principle, a calculation of the critical exponent (more precisely, the critical-exponent matrix) is possible. However, the explicit one-loop renormalization of massive terms in $R^{2}$-gravity with matter has not yet been done. Even if it were known, due to the higher-derivative kinetic terms for the metric, and to the rather nonstandard massive-like terms for the gravitational field, the interpretation of the critical-exponent matrix would still not be clear. These questions require further and careful study, and we hope to return to them elsewhere.

\section{RG-equations in higher-derivative QG with the $O(N)$ $\varphi^{4}$-theory}

In this section we will discuss the RG equations in the $O(N) \varphi^{4}$-model interacting with QG in $D=4-\varepsilon$ dimensions. As we will see, by increasing the number of scalars in the theory, we will be able to decrease the values of the fixed points.

To start with, the Lagrangian of matter is

$$
\mathcal{L}_{m}=\frac{1}{2} g^{\mu \nu} \partial_{\mu} \varphi^{i} \partial_{\mu} \varphi^{i}+\frac{1}{2} \xi R \varphi^{i 2}-\frac{1}{4 !} f \mu^{\varepsilon}\left(\varphi^{i 2}\right)^{2}
$$

where $i=1, \ldots, N$.

For $R^{2}$-gravity interacting with the $O(N)$-theory, the RG equations read

$$
\begin{aligned}
& \frac{d \lambda}{d t}=\beta_{\lambda}=-\varepsilon \lambda-\frac{\alpha^{2} \lambda^{2}}{(4 \pi)^{2}}, \alpha^{2}=\frac{133}{10}+\frac{N}{60}, \\
& \frac{d \omega}{d t}=\beta_{\omega}=-\frac{\lambda}{(4 \pi)^{2}}\left[\frac{10}{3} \omega^{2}+\left(5+\alpha^{2}\right) \omega+\frac{5}{12}+\frac{3 N}{2}\left(\xi-\frac{1}{6}\right)^{2}\right], \\
& \frac{d \xi}{d t}=\beta_{\xi}=\frac{1}{(4 \pi)^{2}} \frac{N+2}{3} f\left(\xi-\frac{1}{6}\right)+\Delta \beta_{\xi}, \\
& \frac{d f}{d t}=\beta_{f}=-\varepsilon f+\frac{1}{(4 \pi)^{2}} \frac{N+8}{3} f^{2}+\Delta \beta_{f},
\end{aligned}
$$

where $\Delta \beta_{\xi}$ and $\Delta \beta_{f}$ are given by (3.6). Numerical study of the fixed points of the system (4.2) for nonzero $\lambda$ and $\varepsilon=1$ yields the results in table 2 .

So, as we see in that table, for $R^{2}$-gravity with many scalars $\left(N \sim 10^{4}\right.$ or larger $)$ there exists an IR stable fixed point where the values of all the coupling constants are less than one. Hence, perturbation theory may be completely trusted around this IR stable fixed point where a second-order phase transition at nonzero temperature may be expected. We have in this case a few saddle points too, where all the values of the couplings are also less than 1, and therefore a first-order phase transition may be expected around these saddle points.

It is interesting to compare the $f^{*}$ corresponding to the IR stable fixed point (when $N=$ 1000000) with the case of no QG. In the latter, we get

$$
\left.\frac{3 \varepsilon(4 \pi)^{2}}{N+8}\right|_{\varepsilon=1, N=1000000}=0.000474
$$




\begin{tabular}{|c|c|c|c|c|c|}
\hline $\bar{N}$ & $\bar{\lambda}$ & $\bar{\omega}$ & $\bar{\xi}$ & $\overline{f f}$ & nature \\
\hline \multirow[t]{8}{*}{$\overline{10000}$} & 0.88 & -2.354 & 0 & 0 & \multirow{2}{*}{ saddle point } \\
\hline & 0.88 & -1.141 & 0.036 & 0.0001 & \\
\hline & 0.88 & -0.003 & 0.163 & 0.0084 & \multirow{4}{*}{$\begin{array}{c}\text { IR stable fixed point } \\
\text { saddle point } \\
",\end{array}$} \\
\hline & 0.88 & -0.003 & 0.164 & 0.0354 & \\
\hline & 0.88 & -0.031 & 0.185 & 0.0049 & \\
\hline & 0.88 & -0.094 & 0.200 & 0.0041 & \\
\hline & 0.88 & -53.135 & 0 & 0 & \multirow[t]{2}{*}{ 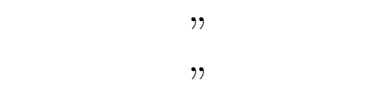 } \\
\hline & 0.88 & -54.896 & 0.082 & 0.0455 & \\
\hline \multirow[t]{8}{*}{100000} & -0.094 & -2.485 & 0 & 0 & \multirow{2}{*}{$\begin{array}{c}\text { saddle point } \\
\text { " }\end{array}$} \\
\hline & -0.094 & -1.450 & 0.039 & 0.000001 & \\
\hline & -0.094 & -0.002 & 0.162 & 0.000216 & \multirow{4}{*}{$\begin{array}{c}\text { IR stable fixed point } \\
\text { saddle point } \\
, "\end{array}$} \\
\hline & -0.094 & -0.001 & 0.164 & 0.004044 & \\
\hline & -0.094 & -0.003 & 0.184 & 0.000051 & \\
\hline & -0.094 & -0.125 & 0.204 & 0.000041 & \\
\hline & -0.094 & -503.005 & 0 & 0 & " \\
\hline & -0.094 & -504.868 & 0.083 & 0.004717 & $"$ \\
\hline \multirow[t]{8}{*}{1000000} & -0.0095 & -2.4985 & 0 & 0 & \multirow{8}{*}{$\begin{array}{c}\text { saddle point } \\
" \\
" \\
\text { IR stable fixed point } \\
\text { saddle point } \\
" \\
" \\
"\end{array}$} \\
\hline & -0.0095 & -1.4540 & 0.039 & 0.000000 & \\
\hline & -0.0095 & -0.0020 & 0.162 & 0.000002 & \\
\hline & -0.0095 & -0.0001 & 0.165 & 0.000439 & \\
\hline & -0.0095 & -0.0266 & 0.183 & 0.000001 & \\
\hline & -0.0095 & -0.1278 & 0.204 & 0.000000 & \\
\hline & -0.0095 & -5002.9915 & 0 & 0 & \\
\hline & -0.0095 & -5004.8753 & 0.083 & 0.000474 & \\
\hline
\end{tabular}

Table 2: Numerical values for the fixed points of the RG equations (4.2). 
(see the figure in the previous table, which gives 0.000439 for the same quantity). As we see, this value is changed (by about a 7\%) in the presence of QG. When $N$ decreases this QG perturbation of the IR stable fixed point gets more and more significant. Thus, the QG perturbation of the IR-stable fixed point is quite a visible effect even at very large $N$. In the absence of QG the critical exponent is quite well-known [9]

$$
\frac{1}{\nu}=2-\frac{N+2}{N+8} \varepsilon+O\left(\varepsilon^{2}\right)
$$

Hence, at large $N$ we obtain the approximate value of the critical exponent even in the presence of QG. It is clear from the above discussion that, for large $N$, QG corrections to this critical exponent will be negligible.

\section{The RG-improved effective potential in three dimen- sions}

As an application of the above results, we will now study the RG-improved effective potential in three dimensions in the presence of QG.

We will consider the $S U(2)$ model. It is known that the IR behaviour of the SM is determined by the confining phase of the $S U(2)$ theory. Thus, these results may be useful for the study of the SM at nonzero temperature. First of all, we present the solution for $f(t)$ when QG is absent (see also [9] and [29]):

$$
f(t)=g^{2}(t)\left\{\frac{\sqrt{2151}}{11} \tan \left[\arctan \left(\frac{\frac{f}{g^{2}}-\frac{15}{11}}{\frac{\sqrt{215}}{11}}\right)-\frac{\sqrt{239}}{14} \log \left(e^{\varepsilon t} \frac{g^{2}(t)}{g^{2}}\right)\right]+\frac{15}{19}\right\}
$$

If QG corrections are small, this solution provides an approximate description of the situation. Going on to cases where QG corrections are significant, we will restrict ourselves to a set-up in which $\lambda(t)$ is proportional to $g^{2}(t)$. This is a very common situation, as there exist many GUT models with $R^{2}$-gravity [21] (see [19] for a review) which are asymptotically free for all coupling constants, and where the QG coupling $\lambda(t)$ is of the same order as the gauge coupling.

One can study (but only numerically) the running coupling constant $f(t)$ for $R^{2}$-gravity. Of course, the above discussion is somewhat speculative, as the way QG corrections show up in the IR region is anything but clear. (Let us recall, however, the proposed models of effective QG in the far infrared [4] which may provide the framework for such large corrections).

We may also examine the situation where the QG corrections are large as compared with scalar loops (gauge coupling corrections are usually so). Then, one can write

$$
\frac{d f}{d t}=-\varepsilon f+\frac{1}{(4 \pi)^{2}}\left(72 g^{4}+\frac{5}{12} \lambda^{2}\right), \quad f(0)=f
$$


which has the following solution:

$$
f(t)=e^{-\varepsilon t}\left\{f-\frac{36 g^{2}}{7}\left[\frac{1}{1-\frac{14 g^{2} x}{\varepsilon}}-1\right]-\frac{5 \lambda}{167}\left[\frac{1}{1-\frac{167 \lambda x}{12}}-1\right]\right\}
$$

where $x=\frac{e^{-\varepsilon t}-1}{(4 \pi)^{2}}$. As one can see explicitly, even for $|\lambda| \simeq g^{2}$ ( $\lambda$ may be positive or negative in (5.3)) QG corrections to $f(t)$ are very small. However, for $\lambda>g^{2}$, QG corrections to (5.3) are still small but now QG influences the dynamics in a different way. Indeed, for $\lambda>g^{2}, \mathrm{QG}$ effects determine the Landau pole, which is reached before the Landau pole associated to $g^{2}(t)$.

Now we may examine the RG-improved potential in the theory under discussion. The standard way to write the $\mathrm{RG}$ equation for the effective potential $V^{\varepsilon}(\varphi)$ is

$$
\left(\mu \frac{\partial}{\partial \mu}+\beta_{p_{i}} \frac{\partial}{\partial p_{i}}-\gamma \varphi \frac{\partial}{\partial \varphi}\right) V^{\varepsilon}(\varphi)=0
$$

(for a recent, similar discussion without QG see [28, [29], [33], and in curved spacetime, see [27]). where $p_{i}$ are all our coupling constants, the $\beta_{p_{i}}$ 's denote their corresponding $\beta$-functions, and $\gamma$ is the anomalous scaling dimension for the scalar field $\varphi$. Working on flat background one easily gets:

$$
V^{\varepsilon}(\varphi)=\frac{\mu^{\varepsilon} f(t)}{4 !} \varphi^{4}(t)
$$

where $\varphi^{2}=\varphi_{a}^{2}, f(t)$ (as well as $g^{2}(t), \lambda(t)$ ) are defined by eqs. (3.12), and $f(t)$ has already been found (see above). The effective field satisfies

$$
\frac{d \varphi(t)}{d t}=-\gamma(t) \varphi(t)
$$

for $R^{2}$-gravity with $S U(2)$ model

$$
\gamma=\frac{1}{(4 \pi)^{2}}\left[-6 g^{2}+\frac{\lambda}{4}\left(\frac{13}{3}-8 \xi-3 \xi^{2}-\frac{1}{6 \omega}-\frac{2 \xi}{\omega}+\frac{3 \xi^{2}}{2 \omega}\right) .\right]
$$

Then,

$$
\varphi(t)=\varphi \exp \left[\int_{0}^{t} d t \gamma(t)\right]
$$

Substituting (5.8) into (5.5), taking $\varepsilon=1$ and using the explicit expressions for the couplings one can find the leading-log behaviour of the effective potential in three dimensions with account of QG corrections. Note that $t=\frac{1}{2} \log \frac{\varphi^{2}}{\mu^{2}}$ and $\mu$ should be identified with the temperature [29]. Examples of the RG-improved potential thus obtained are shown in Fig. 1, for the $S U(2)$ model with and without QG. The appearance of a Landau pole near which perturbation theory breaks down is clearly seen in these figures. 
As one can realize, QG corrections to the $D=3 \mathrm{RG}$-improved potential, even in the $\lambda \simeq g^{2}$ case, are quite small. Here, we have shown how QG corrections in the infrared behaviour of the SM can be in principle taken into account - through the study of the confining phase of the $S U(2)$ theory. Despite the expectations that the IR phase of QG could be important for particle phenomenology in the GUT epoch, we have shown with this simple example that QG corrections are actually not so essential — while they may, of course, still change the numerical values of the quantities obtained in the absence of QG.

\section{Conclusions}

In this paper we have discussed renormalizable higher-derivative QG with matter in $D=4-\varepsilon$ dimensions. The RG equations have been written for $D=4-\varepsilon$ with different choices of matter and the fixed points of these equations have been found. In the example of $f \varphi^{4}$-theory with QG we show, by numerically solving these equations, that the model possesses a rich phase structure, and new fixed points for the scalar coupling constant - with respect to the case of no QG - come into existence. Unfortunately, no new IR stable fixed points appear as a result of QG effects. The IR stable fixed point for the scalar coupling constant, which was already present in the absence of QG, is perturbed (about a $7 \%$ ) by QG effects. Near this IR stable fixed point, it is predicted that the theory at nonzero temperature undergoes a second-order phase transition.

For higher-derivative QG with scalar QED and with nonabelian gauge fields, we obtain only unstable fixed points, as solutions of the RG equations. We have also considered the $O(N) \varphi^{4}$-theory, where gravitational corrections to fixed points get smaller when we increase the number of scalars — as it should be. In this case, the IR stable fixed point, perturbed by QG, exists as well.

We have studied the $S U(2)$ gauge model with scalars interacting with higher-derivative QG and calculated the RG-improved effective potential at nonzero temperature in that theory (which approximately describes the confining phase of the standard model). Numerical estimates show that QG corrrections can give some nonessential contributions to this potential, which can actually become sizeable near the Landau pole (at least for some values of the gravitational coupling $\lambda$ ).

Recently, $R^{2}$-gravity without matter was investigated in $2+\varepsilon$ dimensions [15]. It would be of interest to try and combine the $4-\varepsilon$ and $2+\varepsilon$ approaches in $R^{2}$-gravity, which we have attempted to formulate here, in order to obtain somehow coinciding results at $\varepsilon=1$. Such a synthesis might certainly enrich both approaches very much.

In summary, for the system consisting in renormalizable $R^{2}$-gravity with a scalar-gauge theory near four dimensions, we have shown that the $\epsilon$ expansion technique can be applied to study the critical behavior of the theory and its temperature phase transitions. This model provides an explicitly workable example of how four-dimensional QG could actually influence some well established results [10] on temperature phase transitions and IR fixed points. 


\section{Acknowledgements}

S.D. Odintsov thanks I. Antoniadis and R. Tarrach for discussions. This work has been supported by DGICYT (Spain), project PB93-0035, by CIRIT (Generalitat de Catalunya) and by RFFR, project 94-02-03234. 


\section{References}

[1] G.W. Gibbons, S.W. Hawking and M.J. Perry, Nucl. Phys. B 138 (1978) 141.

[2] L.H. Ford, Phys. Rev. D31 (1985) 710;

I. Antoniadis, J. Iliopoulos and T.N. Tomaras, Phys. Rev. Lett. 56 (1986) 1319;

B. Allen and M. Turyn, Nucl. Phys. B 212 (1988) 147;

T.R. Taylor and G. Veneziano, Phys. Lett. B 212 (1988) 147;

I. Antoniadis and E. Mottola, J. Math. Phys. 32 (1991) 1037;

N.C. Tsamis and R.P. Woodard, Commun. Math. Phys. 162 (1994) 217.

[3] T.R. Taylor and G. Veneziano, Nucl. Phys. B345 (1990) 210.

[4] I. Antoniadis and E. Mottola, Phys. Rev. D 46 (1992) 2013;

S.D. Odintsov, Z. Phys. C54 (1992) 531;

I. Antoniadis, P.O. Mazur and E. Mottola, Nucl. Phys. B 388 (1992) 627;

I. Antoniadis and S.D. Odintsov, Mod. Phys. Lett. A8 (1993) 979.

[5] S. Deser, M.J. Duff and C. Isham, Nucl. Phys. B111 (1976) 45;

N.D. Birrel and P.C. Davies, Quantum Fields in Curved Space-Time, Cambridge Univ. Press, 1982.

[6] J. Zinn-Justin, Quantum field theory and critical phenomena (Oxford Univ. Press, Oxford, 1989).

[7] K.G. Wilson and J. Kogut, Phys. Rep. 12 (1974) 75.

[8] E. Brezin, J.C. Le Guillou and J. Zinn-Justin, in Phase transitions and critical phenomena, Vol. 6, ed. C. Domb and M.S. Green (Academic Press, London, 1989).

[9] K.G. Wilson, Phys. Rev. Lett. 28 (1972) 548;

J.H. Chen, T.C. Lubensky and D.R. Nelson, Phys. Rev. B17 (1978) 4274.

[10] P. Ginsparg, Nucl. Phys. B170 [FS1] (1980) 388.

[11] T. Appelquist and R.D. Pisarski, Phys. Rev. D 23 (1981) 2305.

[12] C. Wetterich and M. Reuter, Nucl. Phys. B408 (1993) 91.

[13] S. Weinberg, in General Relativity, an Einstein Centenary Survey, eds. S.W. Hawking and W. Israel, C.U.P., 1979;

R. Gastmans, R. Kallosh and C. Truffin, Nucl. Phys. B133 (1978) 417;

S.M. Christensen and M.J. Duff, Phys. Lett. B79 (1978) 213;

H. Kawai and N. Ninomiya, Nucl. Phys. B336 (1990) 115;

Y. Tanii, S.-I. Kojima and N. Sakai, Nucl. Phys. B246 (1994) 223;

H. Kawai, Y. Kitazawa and M. Ninomiya, Nucl. Phys. B393 (1993) 280. 
[14] I. Jack and D.R.T. Jones, Nucl. Phys. B358 (1991) 695.

[15] J. Nishimura, S. Tamura and A. Tsuchiya, preprint KEK-TH-394, 1994.

[16] G. t'Hooft and M. Veltman, Ann. Inst. H. Poincaré, A 20 (1974) 69;

S. Deser and P. Nieuwenhuizen, Phys. Rev. D10 (1974) 401;

M.H. Goroff and A. Sagnotti, Nucl. Phys. B266 (1986) 709.

[17] M. Green, J. Schwarz and E. Witten, Superstring theory (Cambrige Univ. Press, Cambridge, 1987).

[18] K.S. Stelle, Phys. Rev. D 16 (1977) 953.

[19] I.L. Buchbinder, S.D. Odintsov and I.L. Shapiro, Effective Action in Quantum Gravity, IOP Publishing, Bristol and Philadephia, 1992).

[20] J. Julve and M. Tonin, Nuovo Cimento B 46 (1978) 137;

E.S. Fradkin and A.A. Tseytlin; Nucl. Phys. B 201 (1982) 469;

I.G. Avramidi, Yad. Fiz. (Sov. J. Nucl. Phys) 44 (1986) 255;

I. Antoniadis and T. Tomboulis, Phys. Rev. D 33 (1986) 2755;

F. Barbero and S.D. Odintsov, Class. Quantum Grav. 10 (1993) 193.

[21] I.L. Buchbinder and I.L. Shapiro, Yad. Fiz. (Sov. J. Nucl. Phys) 44 (1986) 1033;

I.L. Buchbinder, O.K. Kalashnikov, I.L. Shapiro, V.B. Vologodsky and Yu. Yu. Wolfengaut, Phys. Lett. B 216 (1989) 127.

[22] I. Antoniadis, C. Bachas, J. Ellis and D.V. Nanopoulos, Nucl. Phys. B328 (1989) 117;

R.C. Myers, Phys. Lett. B199 (1987) 371.

[23] J. Ambjørn, preprint NBI-HE-94-53 (1994); J. Ambjørn, J. Jurkiewicz and Y. Watabiki, preprint NBI-HE-95-08 (1995).

[24] D. Gross, M.J. Perry and L.G. Yaffe, Phys. Rev. D25 (1982) 330.

[25] F.T. Brandt, J. Frenkel and J.C. Taylor, Nucl. Phys. B374 (1992) 169;

J. Frenkel and J.C. Taylor, Z. Phys. C 49 (1991) 515.

[26] I.L. Buchbinder and S.D. Odintsov, Class. Quantum Grav. 2 (1985) 721.

[27] E. Elizalde and S.D. Odintsov, Phys. Lett. B303 (1993) 240.

[28] C. Ford, D.R.T. Jones, P.W. Stephenson and M. Einhorn, Nucl. Phys. B 395 (1993) 405.

[29] W. Buchmüller and Z. Fodor, Phys. Lett. B331 (1994) 124.

[30] B.L. Voronov and I.V. Tyutin, Yad. Fiz. (Sov. J. Nucl. Phys.) 23 (1976) 664. 
[31] I.L. Buchbinder and S.D. Odintsov, Izw. VUZov. Fiz. (Sov. J. Nucl. Phys.) No 12 (1983) 108 ;

Yad. Fiz. (Sov. Phys. J.) 40 (1984) 1338.

[32] E. Elizalde and S.D. Odintsov, Phys. Lett. B 334 (1994) 33.

[33] M. Bando, T. Kugo, N. Maekawa and H. Nakano, Progr. Theor. Phys. 90 (1993) 405.

[34] D.J. Gross and F. Wilczek, Phys. Rev. Lett. 30 (1973) 1343; H.D. Politzer, Phys. Rev. Lett. 30 (1973) 1346.

[35] N.P. Chang, Phys. Rev. D10 (1974) 2706; T.P. Cheng, E. Eichten and L.F. Li, Phys. Rev. D9 (1974) 2259. 


\section{Figure Captions}

Fig. 1 a) RG-improved effective potentials for the $S U(2)$ model with $\omega(0)=0, \xi(0)=1 / 6$, $g^{2}(0)=0.41, f(0)=0.5$ and $\mu=1$. The three lines drawn correspond to $\lambda(0)=0,0.4$ and 0.8 , as indicated by the labels. b) Same picture for the theory with $\omega(0)=1, \xi(0)=0.5$, and taking $\lambda(0)=10^{-3}, 0.4,0.8$. 
This figure "fig1-1.png" is available in "png" format from: http://arxiv.org/ps/hep-th/9502131v2 
Fig. 1a

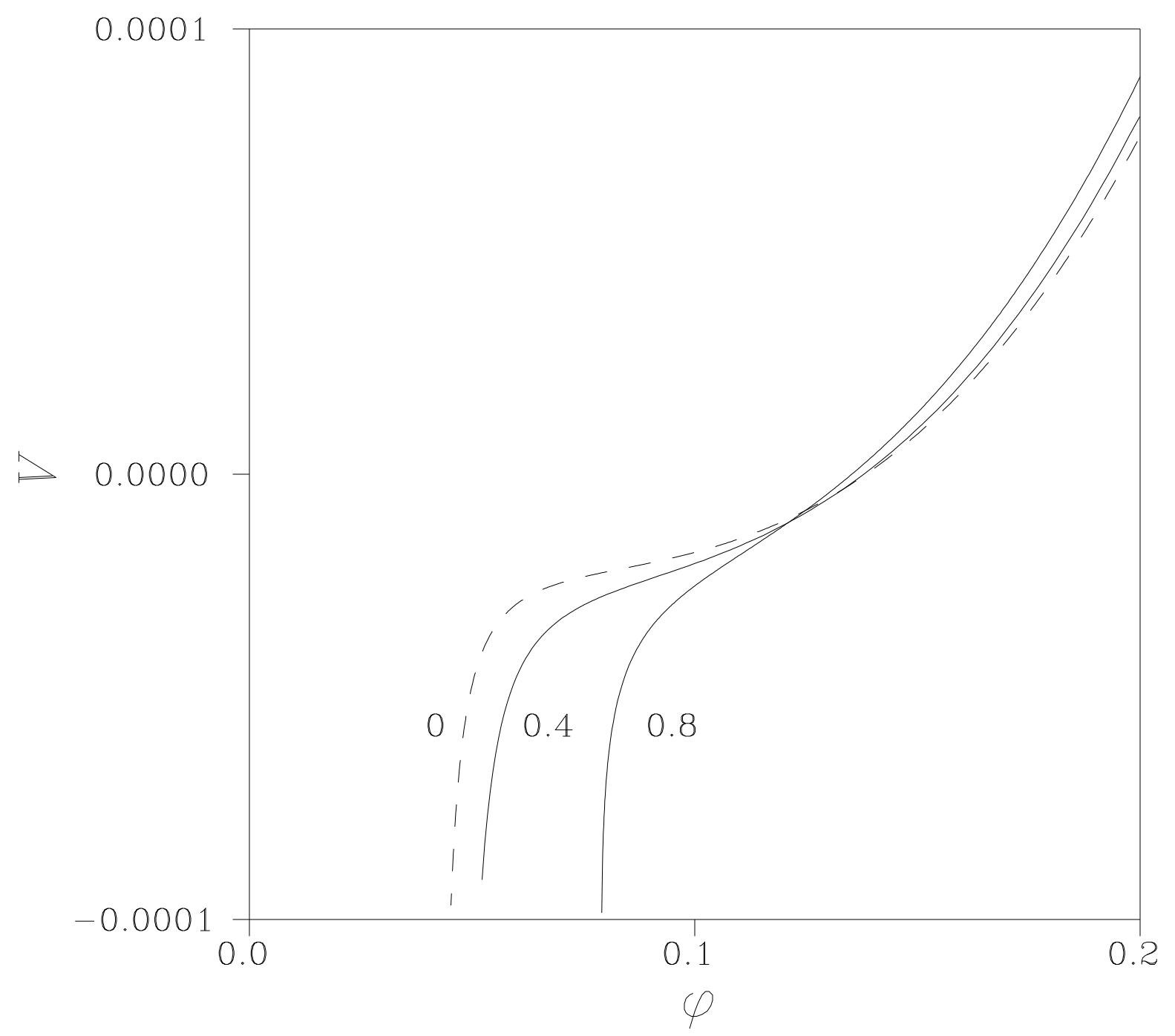



Fig. 1b

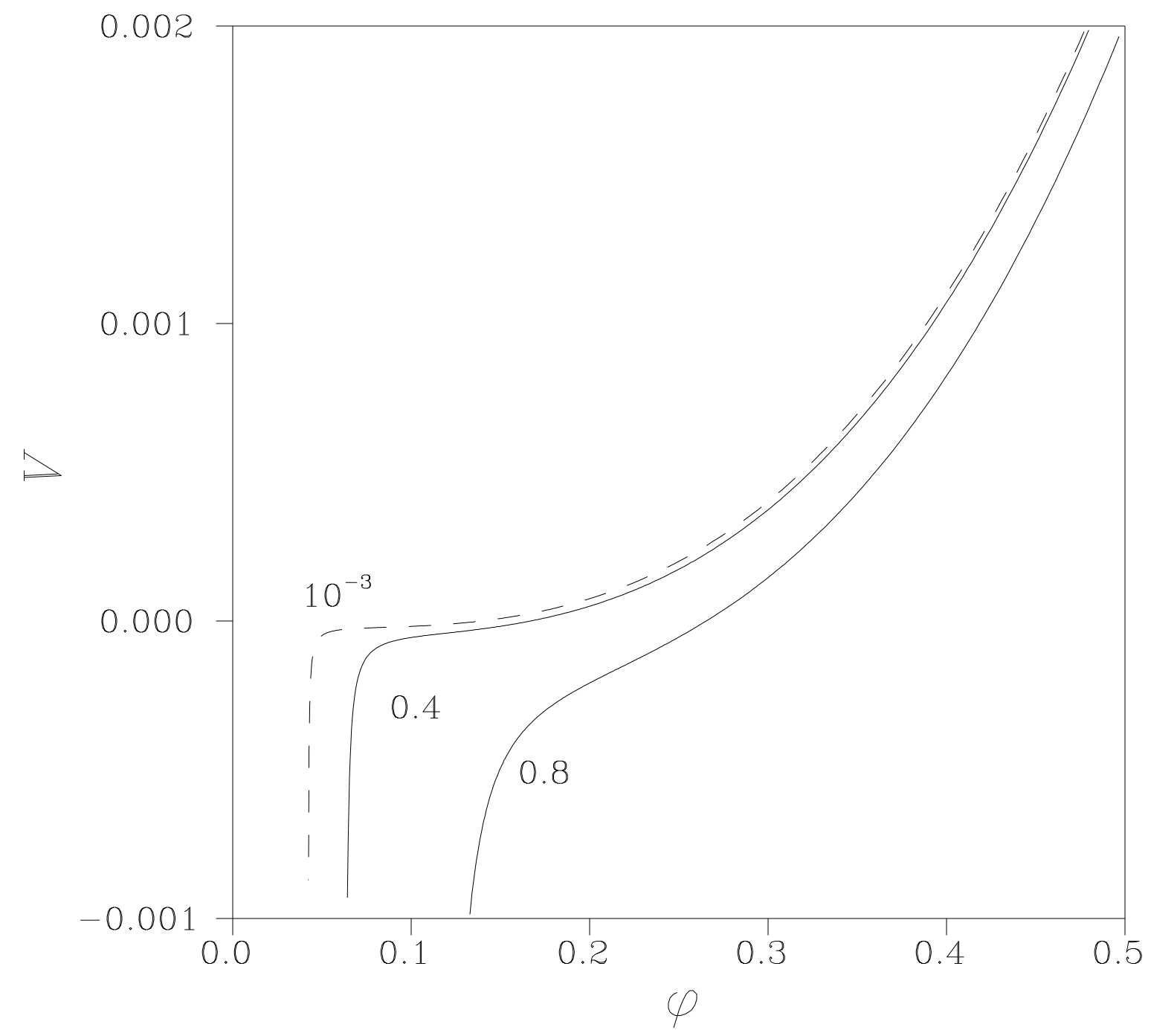



This figure "fig1-2.png" is available in "png" format from: http://arxiv.org/ps/hep-th/9502131v2 
This figure "fig1-3.png" is available in "png" format from: http://arxiv.org/ps/hep-th/9502131v2 